ARTICLE

\title{
Taming interfacial electronic properties of platinum nanoparticles on vacancy-abundant boron nitride nanosheets for enhanced catalysis
}

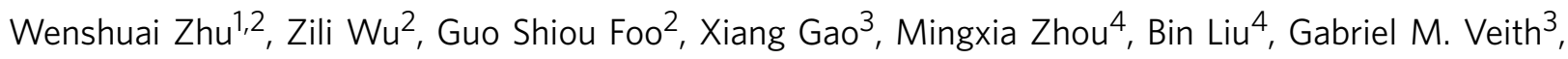
Peiwen Wu ${ }^{1,2}$, Katie L. Browning ${ }^{3}$, Ho Nyung Lee ${ }^{3}$, Huaming $\mathrm{Li}^{5}$, Sheng Dai ${ }^{2}$ \& Huiyuan Zhu ${ }^{2}$

Taming interfacial electronic effects on Pt nanoparticles modulated by their concomitants has emerged as an intriguing approach to optimize Pt catalytic performance. Here, we report Pt nanoparticles assembled on vacancy-abundant hexagonal boron nitride nanosheets and their use as a model catalyst to embrace an interfacial electronic effect on Pt induced by the nanosheets with $\mathrm{N}$-vacancies and B-vacancies for superior $\mathrm{CO}$ oxidation catalysis. Experimental results indicate that strong interaction exists between $\mathrm{Pt}$ and the vacancies. Bader charge analysis shows that with Pt on B-vacancies, the nanosheets serve as a Lewis acid to accept electrons from Pt, and on the contrary, when Pt sits on $\mathrm{N}$-vacancies, the nanosheets act as a Lewis base for donating electrons to Pt. The overall-electronic effect demonstrates an electron-rich feature of Pt after assembling on hexagonal boron nitride nanosheets. Such an interfacial electronic effect makes Pt favour the adsorption of $\mathrm{O}_{2}$, alleviating $\mathrm{CO}$ poisoning and promoting the catalysis.

\footnotetext{
${ }^{1}$ School of Chemistry and Chemical Engineering, Institute for Energy Research, Jiangsu University, Zhenjiang 212013, China. ${ }^{2}$ Chemical Sciences Division, Oak Ridge National Laboratory, Oak Ridge, Tennessee 37831, USA. ${ }^{3}$ Materials Science and Technology Division, Oak Ridge National Laboratory, Oak Ridge, Tennessee 37831, USA. ${ }^{4}$ Department of Chemical Engineering, Kansas State University, Manhattan, Kansas 66506, USA. ${ }^{5}$ Institute for Energy Research, Jiangsu University, Zhenjiang 212013, China. Correspondence and requests for materials should be addressed to S.D. (email: dais@ornl.gov) or to H.Z. (email: zhuh@ornl.gov).
} 
$\mathrm{T}$ uning interfacial electronic properties has emerged as a vital topic in heterogeneous catalysis because of its paramount importance to both practical usage and fundamental understanding of nanoparticle (NP) catalysts ${ }^{1,2}$. Several strategies have been applied to tune the electronic properties of metal nanocatalysts: (1) constructing a multimetallic, that is, binary ${ }^{3-5}$, ternary 6,7 , core/shell ${ }^{8-11}$ or dumbbell structure ${ }^{12}$, where the electronic structure of a catalytically active metal can be modified by other components; (2) using organic ligand modifiers with a desirable electron-donating/withdrawing property on a metal catalyst ${ }^{1,13-16}$; (3) creating metal-support interactions ${ }^{17-21}$, including the strong metal support interaction as well as employing redox-active supports and thereby introducing electronic/geometric modifications on metal catalysts ${ }^{22,23}$. Although the first two strategies have been well-documented in the literature, renewed interest on the metal-support interface has only been witnessed in recent years.

Supported Pt NP catalysts have continuously drawn broad and increasing attention because of their unique catalytic activity for a large number of important chemical reactions, for example, CO oxidation ${ }^{24,25}$, small molecule hydrogenation ${ }^{1}$, oxygen reduction ${ }^{26,27}$ and hydrogen evolution ${ }^{28}$. However, a challenge remains regarding how to arbitrarily tune the electronic structure of $\mathrm{Pt}$ for enhanced catalysis. Taking CO oxidation as an example-on a Pt surface, owing to the strong binding of $\mathrm{CO}$, the $\mathrm{O}_{2}$ activation is usually blocked; therefore a redox-active support $\left(\mathrm{TiO}_{2}\right.$ (ref. 29), $\mathrm{CeO}_{2}$ (refs 30,31), $\mathrm{FeO}_{\mathrm{x}}$ (ref. 32)) that can transfer its lattice oxygen to the Pt catalyst's surface is a prerequisite for efficient $\mathrm{CO}$ oxidation. In the case of a non-redox active support $\left(\mathrm{SiO}_{2}, \mathrm{Al}_{2} \mathrm{O}_{3}\right.$ and $\left.\mathrm{C}\right)$, the $\mathrm{Pt}$ activity for $\mathrm{CO}$ oxidation is usually very low with a full conversion temperature $\left(T_{100}\right)$ above $150^{\circ} \mathrm{C}$ (ref. 32) (Supplementary Table 1). Here our Pt NPs on a non-redox active hexagonal boron nitride ( $h$-BNNs) support with low loading (Pt content $=1.18 \mathrm{wt} \%$ by inductively coupled plasma optical emission spectroscopy determination) can catalyse $\mathrm{CO}$ oxidation with a $T_{100}$ below $70^{\circ} \mathrm{C}$. Meanwhile, there is to our knowledge no report of Pt NPs assembled on vacancy-abundant hexagonal boron nitride nanosheets ( $h$-BNNS) to embrace an interfacial electronic effect on Pt induced by 2-dimensional (2D) $h$-BNNS with $\mathrm{N}$-vacancies $\left(\mathrm{N}_{\mathrm{V}}\right)$ and $\mathrm{B}$-vacancies $\left(\mathrm{B}_{\mathrm{V}}\right)$ for superior $\mathrm{CO}$ oxidation catalysis. Recently, we have reported a gas exfoliation method to prepare ultrathin $h$-BNNS with high surface area and abundant edges and $\mathrm{B} / \mathrm{N}$ vacancies ${ }^{33}$, which could serve as an ideal non-redox active support for Pt NPs. These exfoliated $h$-BNNS are characterised by fewer layers, smaller lateral size and more $\mathrm{B} / \mathrm{N}$ defects including a high exposure of $\mathrm{B}_{\mathrm{V}}$ and $\mathrm{N}_{\mathrm{V}}$ edges than bulk $h$ BN (Fig. 1). Moreover, compared with covalent $\mathrm{C}-\mathrm{C}$ bonding in graphene, the B-N bonding in $h$-BNNS displays partially ionic character, making $h$-BNNS a prominent electronic-tuning support to interact with $\mathrm{Pt}^{23}$.

In this work, we demonstrate an interfacial electronic effect on Pt NP catalysts modulated by the vacancy-abundant $h$-BNNS for superior CO oxidation performance. The Pt NPs supported on $h$-BNNS (denoted as $\mathrm{Pt} / h$-BNNS) demonstrate a high $\mathrm{CO}$ oxidation activity. The durability test shows that the $\mathrm{Pt} / \mathrm{h}$-BNNS catalyst maintains $100 \%$ CO conversion efficiency after $50 \mathrm{~h}$ at $75^{\circ} \mathrm{C}$. The interfacial electronic effect is found to stem primarily from the interaction between $\mathrm{Pt}$ and $\mathrm{B}_{\mathrm{V}}$ as well as Pt and $\mathrm{N}_{\mathrm{V}}$. Both electron energy loss spectroscopy (EELS) and density functional theory (DFT) calculations suggest that there exists a strong interaction between the $\mathrm{Pt}$ and $\mathrm{B} / \mathrm{N}$ vacancies. EELS results indicate that the interaction between $\mathrm{Pt}$ and the $\mathrm{N}_{\mathrm{V}}$ is predominant. According to Bader charge analysis, we find that with $\mathrm{Pt}$ on $\mathrm{B}_{\mathrm{V}}$, the $h$-BNNS serves as a Lewis acid to accept electrons from $\mathrm{Pt}$, whereas when Pt interacts with $\mathrm{N}_{\mathrm{V}}$, the
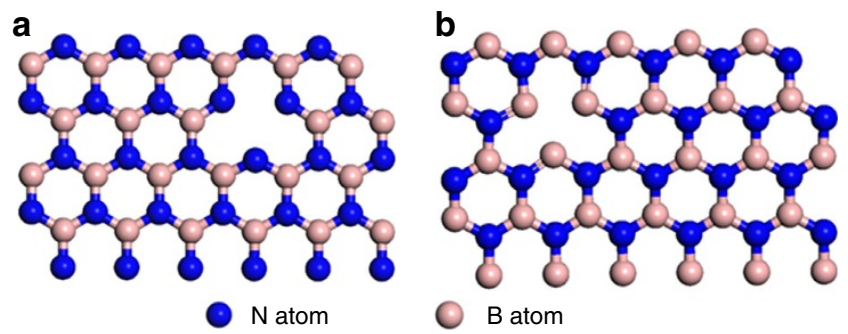

Figure 1 | Schematic illustration of $\boldsymbol{h}$-BNNS with $B$ and $\mathbf{N}$ vacancies. (a) $h$-BNNS with B vacancy and N-terminated edge. (b) $h$-BNNS with $\mathrm{N}$ vacancy and $\mathrm{B}$-terminated edge.

$h$-BNNS acts as a Lewis base for donating electrons to Pt. The overall-electronic effect evaluated by in situ diffuse reflectance infrared spectroscopy (DRIFTS) demonstrates that $\mathrm{Pt}$ is electronic rich with $\mathrm{Pt} / \mathrm{Nv}$ as the primary interaction. Consequently, such an interfacial electronic effect makes $\mathrm{Pt}$ NPs favour the adsorption of $\mathrm{O}_{2}$, alleviating $\mathrm{CO}$ poisoning and promoting the catalysis. Our discovery gives understanding of the interfacial electronic effects between Pt and a non-redox active support and may provide an important strategy to tailor the Pt electronic structure for enhanced catalysis.

\section{Results}

Synthesis and characterization of Pt/ $h$-BNNS. The $h$-BNNS and Pt NPs were synthesized according to previous reports ${ }^{33,34}$. The preparation of $\mathrm{Pt} / h$-BNNS catalyst follows a sonication-assisted colloidal deposition and the process is presented in the Experimental section. Figure 2a and Supplementary Fig. 1 show the representative bright-field transmission electron microscopy (TEM) and scanning transmission electron microscopy (STEM) images of exfoliated few-layer $h$-BNNS with high exposure edge and $5 \pm 0.5 \mathrm{~nm}$ cubic Pt NPs. X-ray diffraction pattern indicates that the Pt NPs have the face-centred cubic structure (Supplementary Fig. 2), and the size estimated from Scherrer equation is consistent with the results from TEM and STEM observations. The (111) peak is much stronger than the (200) peak, demonstrating a three-dimensional randomly oriented assembly.

Bright-field TEM (Fig. 2a) and STEM (Fig. 2b) images of the $\mathrm{Pt} / h$-BNNS demonstrate that the cubic Pt NPs embedded at $h$-BNNS sitting mostly on the edge vacancies of the support. As seen by TEM and STEM, $h$-BNNS present an ultra-thin-layered structure with abundant edges formed during exfoliation. This ultrathin feature is also proved by atomic force microscopy (AFM) (Supplementary Fig. 3) and the height of $h$-BNNS is 2-3 nm. More importantly, abundant defects consisting of either $\mathrm{N}_{\mathrm{V}}$ or $\mathrm{B}_{\mathrm{V}}$ were observed by high-resolution STEM (Fig. 2c). To investigate the interaction between Pt NPs and $h$-BNNS, we performed EELS to map the individual $\mathrm{B}$ and $\mathrm{N}$ in the $\mathrm{Pt} / h$-BNNS sample, observing a chemical shift of B-K edge in its pre-peak depending only on the distance to the $\mathrm{Pt}$ particles (Supplementary Fig. 4 and Supplementary Movie 1). The B-K edge profiles acquired from the $h$-BNNS matrix region (2) and from the centre of the $\mathrm{Pt} / h$-BNNS overlapping region (1) are shown in Fig. 3a. Compared with the B-K edge obtained from the (2) site, we find that the pre-peak $\left(\pi^{*}\right)$ of B-K downshifts at the (1) site, indicating that there are B-terminated $\mathrm{N}_{\mathrm{V}}$ defects interacting with the Pt NPs (Fig. 3b) ${ }^{35}$. Supplementary Movie 1 further proves that this downshift of the pre-peak of B-K is ubiquitous depending only on the distance to Pt NPs. This is consistent with the formation of an additional peak at the threshold of N-K edge at (2) site ${ }^{36}$, as shown by the arrow in Fig. 3c. The additional peak appears relatively weak, although 

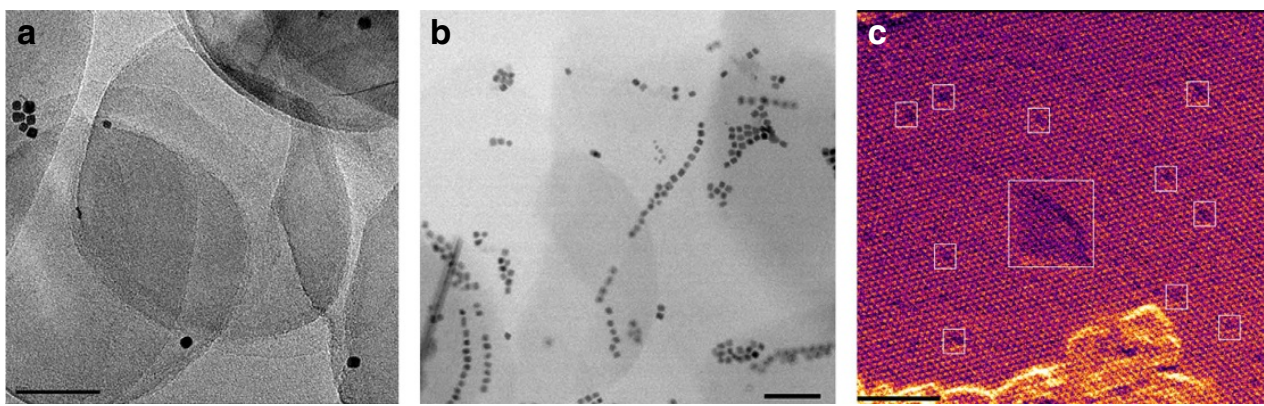

Figure 2 | Representative TEM and STEM images. (a) TEM of Pt/h-BNNS. Scale bar, $50 \mathrm{~nm}$. (b) STEM of Pt/h-BNNS. Scale bar, $50 \mathrm{~nm}$. (c) Highresolution STEM of $h$-BNNS with vacancies, as indicated by the boxes. Scale bar, $2 \mathrm{~nm}$.
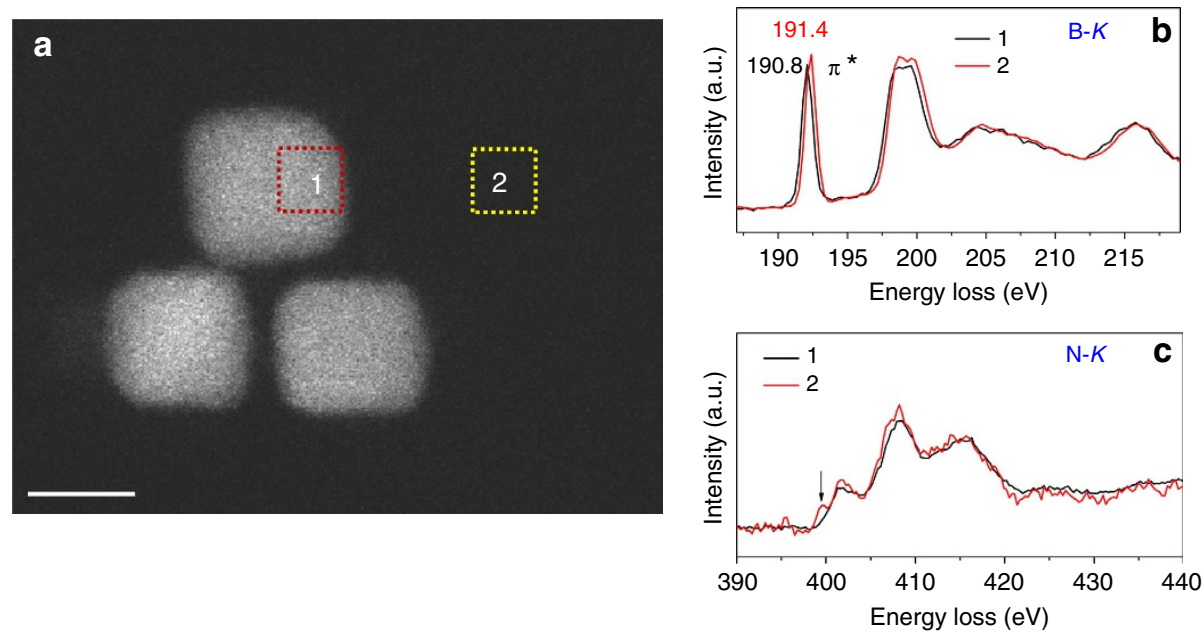

Figure 3 | EELS analysis of Pt/h-BNNS. (a) Annular dark-field (ADF) STEM image of Pt/h-BNNS indicating the mapping region: 1. The centre of $\mathrm{Pt} / h$-BNNS overlapping region; 2. h-BNNS matrix. (b) B-K EELS profile and (c) N-K EELS profile mapped from regions 1 and 2.

after careful examination we did not observe it in the pure $\mathrm{BN}$ matrix region. These results indicate that Pt NPs may prefer to be embedded on B-terminated $\mathrm{N}_{\mathrm{V}}$ of $h$-BNNS instead of staying on $\mathrm{N}$-terminated $\mathrm{B}_{\mathrm{V}}$ of $h$-BNNS.

Catalytic activity for $\mathrm{CO}$ oxidation. The catalytic oxidation of $\mathrm{CO}$ as a probe reaction was evaluated over as-prepared catalysts in a temperature-programmed mode. The catalysts were packed into a U-shaped quartz tube (inner diameter $=4 \mathrm{~mm}$ ), sealed by quartz wool and treated at $200^{\circ} \mathrm{C}$ for $1 \mathrm{~h}$ to activate the surface. CO oxidation was then performed with a gas stream consisting of $1 \%$ CO (balance air, <4 p.p.m. water) at a space velocity (SV) of $1667 \mathrm{Lg}^{-1} \mathrm{Pt}^{-1}$. As shown in Fig. 4a, the $\mathrm{Pt} / h$-BNNS (1.18 wt.\% Pt content, $h$-BNNS $278 \mathrm{~m}^{2} \mathrm{~g}^{-1}$ ) displays the highest CO oxidation activity and readily converts $100 \%$ CO starting from $67^{\circ} \mathrm{C}$. As a comparison, Pt NPs on various non-redox active supports with the similar $\mathrm{Pt}$ content (1.15-1.21 wt.\%), including bulk $h$-BN $\left(10 \mathrm{~m}^{2} \mathrm{~g}^{-1}\right)$, silica fumed $\left(\mathrm{SiO}_{2}, 280 \mathrm{~m}^{2} \mathrm{~g}^{-1}\right)$ and acetylene black $\left(\mathrm{C}, 260 \mathrm{~m}^{2} \mathrm{~g}^{-1}\right)$ are also investigated and their corresponding $T_{100}$ is 104,156 and $128^{\circ} \mathrm{C}$ at a similar SV, respectively. Even with the redox-active support $\mathrm{TiO}_{2}\left(\mathrm{P}_{25}, 65 \mathrm{~m}^{2} \mathrm{~g}^{-1}\right)$, the $T_{100}$ was found to be $88^{\circ} \mathrm{C}$ at a similar SV. This catalytic performance indicates that $h$-BNNS can serve as an excellent support for Pt NPs. This CO oxidation activity is also much higher than the reported redox-active support $\mathrm{TiO}_{2}$-supported $\mathrm{Pt}$ nanocrystals with a $T_{100}$ of $100^{\circ} \mathrm{C}$ and non-redox active support $\mathrm{Pt} / \mathrm{SiO}_{2}$ with a $T_{100}$ of $150^{\circ} \mathrm{C}^{24,37,38}$. The $\mathrm{Pt} / h$-BNNS exhibits negligible CO oxidation activity only at temperatures below $35^{\circ} \mathrm{C}$, which is much lower than that of the active support $\mathrm{TiO}_{2}\left(69^{\circ} \mathrm{C}\right)$ and the inert supports $\mathrm{SiO}_{2}\left(95^{\circ} \mathrm{C}\right)$ and $\mathrm{C}\left(75^{\circ} \mathrm{C}\right)$ under the same reaction conditions. The apparent activation energies $\left(E_{\mathrm{a}}\right)$ are derived with a SV of $10^{4} \mathrm{Lg}^{-1} \mathrm{Pt}^{-1}$ on $\mathrm{Pt} / h$-BNNS and $\mathrm{Pt} / \mathrm{SiO}_{2}$. $\mathrm{Pt} / h$-BNNS has an $E_{\mathrm{a}}$ value of $38.0 \mathrm{~kJ} \mathrm{~mol}^{-1}$, lower than that of the inert support $\mathrm{SiO}_{2}\left(49.3 \mathrm{~kJ} \mathrm{~mol}^{-1}\right)$ (Fig. 4b). For long-time stability study, a time-on-stream test was performed on the $\mathrm{Pt} / h$-BNNS, and the results in Supplementary Fig. 5 indicated that the catalyst maintained $100 \% \mathrm{CO}$ conversion efficiency after $50 \mathrm{~h}$ at $75^{\circ} \mathrm{C}$. 75,50 and $25 \% \mathrm{CO}$ conversions over the $\mathrm{Pt} / h$-BNNS were also achieved at 49,45 and $39^{\circ} \mathrm{C}$ after 15,20 and 15 h test.

Interfacial electronic properties of $\mathbf{P t} / \boldsymbol{h}$-BNNS. To prove the $\mathrm{Pt} / h$-BNNS interface impact on the catalytic activity, we performed measurements of Pt NPs and $h$-BNNS alone under the same reaction conditions (Supplementary Fig. 6) and found $h$-BNNS has no catalytic activity for CO oxidation under $200^{\circ} \mathrm{C}$. The Pt NPs exhibit negligible CO oxidation activity at temperatures below $150^{\circ} \mathrm{C}$ and converts $100 \%$ CO starting from $229^{\circ} \mathrm{C}$, which is much higher than that of $\mathrm{Pt} / h$-BNNS. These results indicate that the enhancement effect is from the interfacial coupling between $\mathrm{Pt}$ and $h$-BNNS. To further understand how the $\mathrm{Pt} / \mathrm{h}$-BNNS interface impacts on the catalytic activity, periodic DFT calculations, employing the Generalized Gradient Approximation-PBE functional, were performed to optimize the $\mathrm{Pt}_{4}$ particle structures, and analyse the charge transfers at the interface of $\mathrm{Pt} / h$-BNNS using Bader charge analysis on pristine 

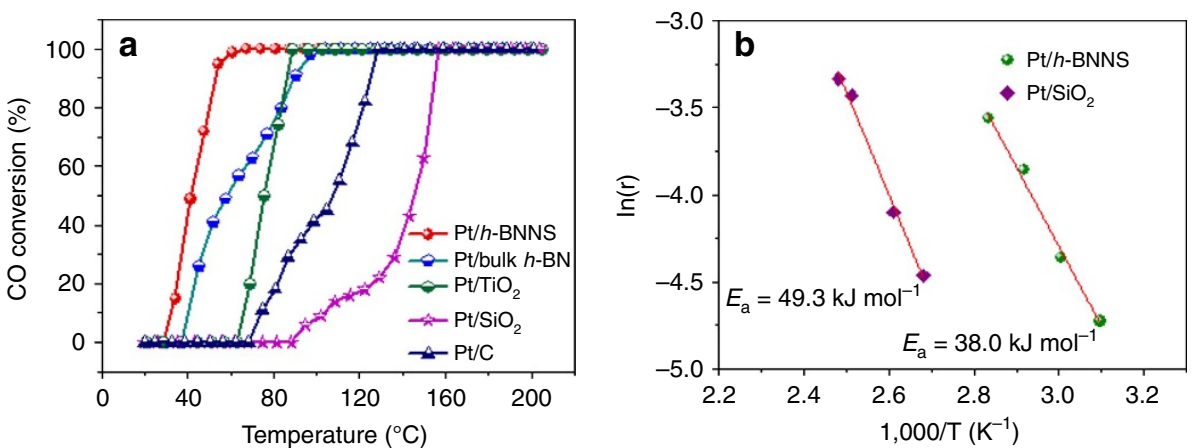

Figure 4 | $\mathbf{C O}$ oxidation activity of Pt/h-BNNS. (a) $\mathrm{CO}$ oxidation light-off curves for the $\mathrm{Pt} / h$ - $\mathrm{BNNS}, \mathrm{Pt} / \mathrm{bulk} h-\mathrm{BN}, \mathrm{Pt} / \mathrm{TiO} 2, \mathrm{Pt} / \mathrm{SiO}{ }_{2}$ and $\mathrm{Pt} / \mathrm{C}$, $\mathrm{m}$ (catalyst) $=30 \mathrm{mg}$, CO flow rate $10 \mathrm{ml} \mathrm{min}{ }^{-1}$. (b) The apparent activation energies $\left(E_{\mathrm{a}}\right)$ of $\mathrm{Pt} / \mathrm{h}-\mathrm{BNNS}$ and $\mathrm{Pt} / \mathrm{SiO}_{2}, \mathrm{~m}(\mathrm{catalyst})=5 \mathrm{mg}, \mathrm{CO}$ flow rate $10 \mathrm{ml} \mathrm{min}^{-1}$.

$h$-BNNS, and $h$-BNNS with $\mathrm{Nv}$ and Bv single-point defects. The optimized geometries and calculated valence electrons on Pt and nearby $\mathrm{B}$ and $\mathrm{N}$ atoms are shown in Fig. 5. Other geometries (for example, flat $\mathrm{Pt}_{4}$ ) were also considered and are presented in Supplementary Figs 7 and 8 . The 4 -atom $\mathrm{Pt}_{4}$ cluster prefers a 3D tetrahedral geometry on $h$-BNNS. On the pristine h-BNNS, the charge transfer between $\mathrm{Pt}$ and $h$-BNNS is negligible as shown in Fig. 5a. The cluster binding energy $(\mathrm{BE})$ is $-2.00 \mathrm{eV}$. In the presence of $\mathrm{Nv}$ and $\mathrm{Bv}$, the cluster preferentially binds at the defective sites, with respective $\mathrm{BEs}$ of $-7.22 \mathrm{eV}$ and $-7.77 \mathrm{eV}$, by forming corresponding $\mathrm{Pt}-\mathrm{B}$ and $\mathrm{Pt}-\mathrm{N}$ bonds. The increase of BEs clearly indicates that vacancy sites have the role of enhancing the adsorptions and stabilising $\mathrm{Pt}$ particles for $\mathrm{CO}$ oxidation. Bader charge analyses confirmed that there exists an interfacial charge transfer between the adsorbed cluster and $h$-BNNS. At the $\mathrm{N}_{\mathrm{V}}$ site (B termination) (Fig. 5b), charge transfers from $h$-BNNS to $\mathrm{Pt}$, resulting in a net gain of 0.80 e on the Pt-1 atom; whereas at the $\mathrm{B}_{\mathrm{V}}$ site $(\mathrm{N}$ termination, Fig. $5 \mathrm{c}$ ), charge transfers away from $\mathrm{Pt}$, resulting in a net loss of $0.72 \mathrm{e}$ on the Pt- 4 atom. A larger $\mathrm{Pt}_{10}$ cluster, with an optimized tetrahedral geometry was also considered, and Bader charge analyses showed that the Pt atoms at the defect sites would also gain and lose net charges at the $\mathrm{Nv}$ and Bv point defect sites, respectively (Supplementary Fig. 9).

On the $\mathrm{Pt}_{4}$ cluster supported on the defect-free $h$-BNNS, CO binds at the top site (Fig. $6 \mathrm{a}$ ), with a $\mathrm{BE}_{(\mathrm{CO})}$ of $-2.65 \mathrm{eV}$. On the cluster adsorbed at the $\mathrm{Bv}$ defective site (charge transferred away from $\mathrm{Pt}$ ), $\mathrm{CO}$ binds in its most stable configuration at the Pt-3 atom, with a $\mathrm{BE}$ of $-2.59 \mathrm{eV}$ (Fig. $6 \mathrm{~b}$ ), weaker than that on the pristine $\mathrm{Pt} / h$-BNNS. In contrast, $\mathrm{CO}$ binds more strongly on the cluster supported on the $h$-BNNS with $\mathrm{Nv}$, with a $\mathrm{BE}$ of $-2.83 \mathrm{eV}$ than on the pristine $h$-BNNS (Fig. $6 \mathrm{c}$ ). Moreover, the BEs of $\mathrm{O}_{2}$ on the $\mathrm{Pt}_{4}$ cluster are stronger with both $\mathrm{Bv}$ $(-2.90 \mathrm{eV})$ (Fig. 6e) and $\mathrm{Nv}(-3.08 \mathrm{eV})$ (Fig. 6f) than that on the defect-free Pt/h-BNNS $(-2.26 \mathrm{eV})$ (Fig. 6d). The optimized geometries and $\mathrm{BEs}$ of $\mathrm{CO}$ and $\mathrm{O}_{2}$ on $\mathrm{Pt}_{10}$ tetrahedral cluster are also considered on pristine $h$-BNNS, as well as $h$-BNNS with Nv and $\mathrm{Bv}$ point defects (Supplementary Fig. 10). The structural deformation on this $\mathrm{Pt}_{10}$ cluster is noticeably to a reduced extent. $\mathrm{CO}$ adsorbs on the top of the $\mathrm{Pt}$ atom in the edge of the cluster supported on the pristine $h$-BNNS and that with the Nv point defect. With the Bv defect, $\mathrm{CO}$ binds on the bridge site closer to the cluster and $h$-BNNS interface. It is also noted that the $\mathrm{BE}_{(\mathrm{CO})}$ increases near the Nv defect, accompanied by net charge gain on the cluster, whereas the $\mathrm{BE}_{(\mathrm{CO})}$ decreases near the $\mathrm{Bv}$ defect, accompanied by net charge loss on the cluster. $\mathrm{O}_{2}$ prefers to adsorb at the bridge site along the cluster edge. The BEs of $\mathrm{O}_{2}$ adsorptions are stronger regardless of the types of vacancy. The trends for both $\mathrm{CO}$ and $\mathrm{O}_{2}$ adsorption are consistent with the findings on smaller clusters. Hence, first-principles calculations support the fact that the electronic effects on $\mathrm{Pt}$ induced by vacancy-abundant $h$-BNNS could faciliate $\mathrm{O}_{2}$ adsorption and activation, thus enhance $\mathrm{CO}$ oxidation, which is in accordance with the findings by DFT calculations in the previous reports 39,40 . This observation also explains why bulk $h$ BN (with smaller surface area $10 \mathrm{~m}^{2} \mathrm{~g}^{-1}$, less edges and vacancies) performs inferior to $h$-BNNS $\left(278 \mathrm{~m}^{2} \mathrm{~g}^{-1}\right.$, abundant of edges and vacancies) ${ }^{33}$ (Supplementary Fig. 11).

\section{Discussion}

As mentioned above, the charge transfer was from $h$-BNNS to $\mathrm{Pt}$ on $\mathrm{Nv}$ and the corresponding opposite direction on Bv. Since both $\mathrm{Nv}$ and $\mathrm{Bv}$ are present in $h$-BNNS, to characterize the overall charge transfer between Pt and $h$-BNNS, in situ DRIFTS of CO adsorption was employed to probe the surface electronic state of $\mathrm{Pt} / h$-BNNS and $\mathrm{Pt} / \mathrm{SiO}_{2}$ (non-redox active support). The in situ DRIFTS spectra in Fig. 7 show that the linearly adsorbed CO on $\mathrm{Pt} / h$-BNNS gives an absorption at $2,075 \mathrm{~cm}^{-1}, \sim 15 \mathrm{~cm}^{-1}$ lower than that on $\mathrm{Pt} / \mathrm{SiO}_{2}$. This redshift of the $\mathrm{CO}$ frequency indicates that the surfaces of $\mathrm{Pt}$ NPs are more negatively charged on $h$-BNNS than on $\mathrm{SiO}_{2}$. It suggests that the overall electron transfer is from $h$-BNNS to $\mathrm{Pt} \mathrm{NPs}^{41}$, consistent with our EELS result that Pt preferably interacts with $B$ atoms at $N_{V}$, where $\mathrm{B}$ atoms at $\mathrm{N}_{\mathrm{V}}$ tend to transfer charge to $\mathrm{Pt}(0.8 \mathrm{eV})$ based on Bader charge analysis (Fig. 5b). X-ray photoelectron spectroscopy (XPS) was performed to probe the change in the $\mathrm{BE}$ of $\mathrm{Pt}$ to further investigate the charge transfer between $\mathrm{Pt}$ and $h$-BNNS. XPS data were collected for the Pt/h-BNNS catalyst in Supplementary Fig. 12. The data showed typical $h$-BNNS spectra with little to no surface oxidation evident in the N1s and B1s plots. This also indicates that the catalytic activity enhancement is not due to the creation of B-O functionality at the support-Pt interface. The $P t 4 f_{7 / 2}$ data show the presence of two unique $P t$ species with BEs of 70.73 and $72.56 \mathrm{eV}$ corresponding to $\mathrm{Pt}^{\mathrm{O}}$ and $\mathrm{Pt}^{2+}$, respectively, with a relative ratio of $4: 1$. The $\mathrm{Pt} 4 \mathrm{f}_{5 / 2}$ data are shifted by $3.3 \mathrm{eV}$ relative to the $\mathrm{Pt} 4 \mathrm{f}_{7 / 2}$ value consistent with the presence of two $\mathrm{Pt}^{\mathrm{O}}$ and $\mathrm{Pt}^{2+}$ species (relative ratio of 3.9:1). This suggests that the majority of the $\mathrm{Pt}$ is in the reduced state consistent with what is required for $\mathrm{CO}$ oxidation. XPS data collected for the non-redox active support of $\mathrm{Pt} / \mathrm{SiO}_{2}$ sample also indicated a signal for the $\mathrm{Pt}$ species consistent with $\mathrm{Pt}^{0}$ and $\mathrm{Pt}^{2+}$ species. However, the corresponding relative ratio of $\mathrm{Pt}^{0}$ and $\mathrm{Pt}^{2+}$ is $0.36: 1$ based on $\mathrm{Pt} 4 \mathrm{f}_{7 / 2}$ and $0.58: 1$ based on Pt $4 \mathrm{f}_{5 / 2}$ as shown in Supplementary Fig. 13, suggesting that $\mathrm{Pt}$ NPs are more negatively charged on h-BNNS than those on $\mathrm{SiO}_{2}$ support owing to the interfacial charge transfer between Pt and $h$-BNNS with vacancies. Meanwhile, the $\mathrm{Pt}^{0} \mathrm{BE}$ in $\mathrm{Pt} / h$-BNNS shifted negatively by $0.32 \mathrm{eV}$ compared with that 


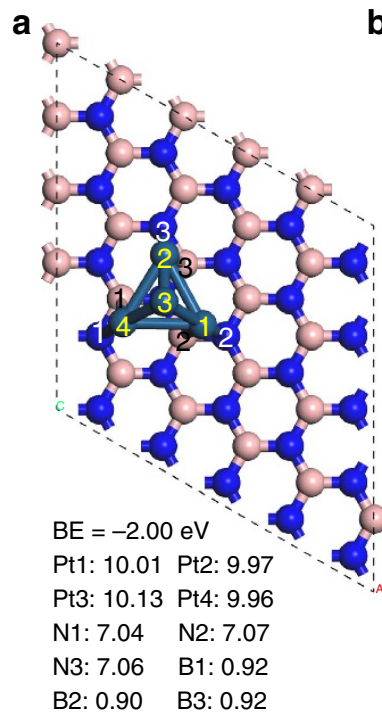

B2: 0.90 B3: 0.92 b

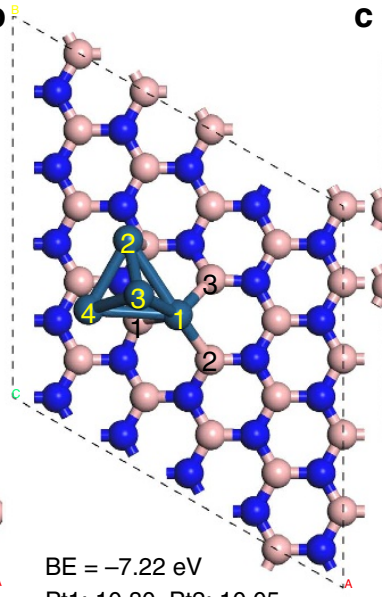

Pt1: 10.80 Pt2: 10.05

Pt3: 10.22 Pt4: 10.05

B1: 1.19 B2: 1.28

B3: 1.21

C

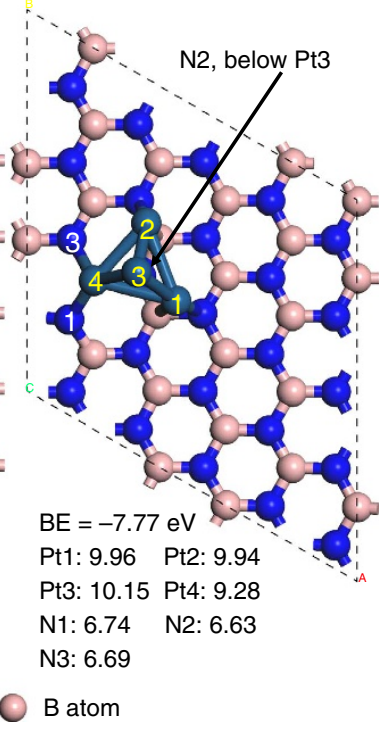

Figure 5 | Optimized structures and valence electrons of pyramidal $\mathbf{P t}_{\mathbf{4}}$ cluster on $\boldsymbol{h}$-BNNS. (a) $\mathrm{Pt}_{4}$ cluster on clean, vacancy-free $h$-BNNS. (b) $\mathrm{Pt}_{4}$ cluster $h$-BNNS with Nv. (c) $\mathrm{Pt}_{4}$ cluster on $h$-BNNS with Bv.

of the $\mathrm{Pt} / \mathrm{SiO}_{2}$, demonstrating $\mathrm{Pt}$ is electron enriched in $\mathrm{Pt} / h$-BNNS ${ }^{42}$.

In summary, we have discovered the interfacial electronic effects between Pt and a non-redox active vacancy-abundant $h$-BNNS support. We found that the Pt NPs were embedded on both Nv and $\mathrm{Bv}$ and the overall charge transfer is from $h$-BNNS to $\mathrm{Pt}$. Consequently, $\mathrm{O}_{2}$ binds stronger than $\mathrm{CO}$ molecules, alleviating the CO poisoning of the Pt NPs and promoting the catalysis. This finding may provide a way to design and develop highly stable supported Pt catalysts with controllable activity and selectivity for other heterogeneous catalysis.

\section{Methods}

Preparation of $\boldsymbol{h}$-BNNS. In a typical preparation process of $h$-BNNS, the commercial $h$-BN (parent bulk $h$-BN, lateral size $1 \mu \mathrm{m}$ ) powder $(1 \mathrm{~g})$ in quartz boat was ramped to $800^{\circ} \mathrm{C}$ in a muffle furnace under air, kept at this temperature for 5 min, and then immediately immersed into a dewar bottle containing liquid $\mathrm{N}_{2}$ $\left(\mathrm{L}-\mathrm{N}_{2}\right)$ until the $\mathrm{L}-\mathrm{N}_{2}$ gasified completely. The above steps were performed repeatedly. The resulting $h$-BNNS was collected by the following process: the as-prepared h-BNNS were dispersed in isopropanol and sonicated for $30 \mathrm{~min}$. Then the dispersion was centrifuged at 800 r.p.m. for $10 \mathrm{~min}$ to remove any remaining bulk crystals. The supernatant was collected and dried in vacuum oven overnight.

Preparation of Pt NPs. Pt (acac) $2(0.1 \mathrm{~g})$, 1-octadecene $(10 \mathrm{ml})$, oleic acid $1 \mathrm{ml}$ and oleylamine $(1 \mathrm{ml})$ were mixed under $\mathrm{N}_{2}$ and magnetic stirring. The mixture was then heated to $65^{\circ} \mathrm{C}$ to dissolve $\mathrm{Pt}(\mathrm{acac})_{2}$. The temperature was then raised to $\sim 160^{\circ} \mathrm{C}$. A solution of $\mathrm{Fe}(\mathrm{CO})_{5}$ in hexane $(0.1 \mathrm{ml}$, prepared by adding $0.1 \mathrm{ml}$ $\mathrm{Fe}(\mathrm{CO})_{5}$ in $1 \mathrm{ml}$ hexane under $\mathrm{N}_{2}$ ) was injected into the hot solution under $\mathrm{N}_{2}$ blanket. The solution was further heated to $200^{\circ} \mathrm{C}$ and kept at this temperature for $1 \mathrm{~h}$ before it cooled down to room temperature. In total, $40 \mathrm{ml}$ of isopropanol was added and then suspension was centrifuged $(8,000$ r.p.m., $\sim 10 \mathrm{~min})$ to separate the Pt NPs. The particles were dispersed in $10 \mathrm{ml}$ hexane and precipitated out by adding ethanol. The process was repeated one more time to purify the NPs. The as-prepared product was dispersed in $10 \mathrm{ml}$ hexane for next use.

Preparation of Pt/h-BNNS catalysts. A certain volume of Pt NPs hexane solution was blow-dried by $\mathrm{N}_{2}$, weighing the mass of Pt NPs. A certain amount of $h$-BNNS (mass ratio of Pt NPs and $h$-BNNS is 1:12) was dispersed in the mixture of ethanol $(5 \mathrm{ml})$ and hexane $(5 \mathrm{ml})$ under sonication. Pt NPs were re-dispersed in the hexane $(5 \mathrm{ml})$ under sonication and dropped slowly into the $h$-BNNS solution, sonicating for $1 \mathrm{~h}$. The as-prepared sample was separated via centrifugation, washed with ethanol three times and dried in vacuum at $50^{\circ} \mathrm{C}$ for further use. Pt content was 1.18 wt.\% by inductively coupled plasma optical emission spectroscopy determination. The details for the preparation of $\mathrm{Pt} / \mathrm{bulk} h$-BN, $\mathrm{Pt} / \mathrm{TiO}_{2}, \mathrm{Pt} / \mathrm{SiO}_{2}$ and $\mathrm{Pt} / \mathrm{C}$ catalysts were presented in supplementary information.
Catalyst characterisation. AFM was performed on Cypher AFM (Asylum Research) equipped with Nanosensor PPP-EFM probes, which typically have a moderate stiffness of $2-5 \mathrm{~N} \mathrm{~m}^{-1}$; TEM was performed using a Zeiss Libra 120 system operating at $120 \mathrm{kV}$. Quantitative elemental analysis was done on an inductively coupled plasma optical emission spectrometry on a Perkin-Elmer Optima 2000 DV ICP spectrometer. XPS data were collected using a PHI 3056 spectrometer with an $\mathrm{Al}$ anode source operated at $15 \mathrm{kV}$ and an applied power of $350 \mathrm{~W}$ with samples mounted on indium foil. The higher energy species in the Pt data corresponds to Indium $4 \mathrm{p}$ from the substrate (onset $78 \mathrm{eV}$ ), which were unavoidable due to the samples adhesion to the indium foil. Annular dark-field imaging and EELS analysis were carried out using a Nion UltraSTEM100 operated at $60 \mathrm{kV}$ under ultrahigh vacuum $\left(1 \times 10^{-9}\right.$ torr $)$. The microscope is equipped with a cold field-emission gun and an aberrations corrector for sub-angstrom resolution. An inner angle no smaller than $30 \mathrm{mrad}$ was used for annular dark-field imaging. X-ray diffraction was performed on a Panalytical Empyrean diffractometer with $\mathrm{Cu} \mathrm{K}$-alpha radiation $\lambda=1.5418 \AA$ operating at $45 \mathrm{kV}$ and $40 \mathrm{~mA}$. In situ DRIFTS measurement was performed using a Nicolet Nexus 670 spectrometer equipped with a MCT detector cooled by liquid nitrogen. Each spectrum was recorded with 32 scans at a resolution of $4 \mathrm{~cm}^{-1}$. The sample was loaded in a porous ceramic cup and inserted into an in situ chamber (HC-900, Pike Technologies). The catalyst was pre-treated in the chamber at $200^{\circ} \mathrm{C}$ for $1 \mathrm{~h}$ in flowing $5 \% \mathrm{O}_{2} / \mathrm{He}$ before cooling down to room temperature for $\mathrm{CO}$ adsorption.

General procedure for $\mathrm{CO}$ oxidation. $\mathrm{CO}$ oxidation was carried out in a temperature-controlled microreactor (Altamira AMI 200) equipped with an on-line gas chromatograph. All experiments were performed under atmospheric pressure with a flow rate of $10 \mathrm{ml} \mathrm{min}^{-1}$ of $1 \%$ CO balanced in dry air. For the kinetic measurements, the amount of $\mathrm{Pt} / h$-BNNS and $\mathrm{Pt} / \mathrm{SiO}_{2}$ was reduced to $5 \mathrm{mg}$, respectively, to ensure the $\mathrm{CO}$ conversion is below $15 \%$. The $\mathrm{CO}$ conversion was averaged at 10,20,30 and $40 \mathrm{~min}$ to calculate the reaction rate. The reaction rate $(r)$ was calculated as equation (1):

$$
r=\text { CO conversion rate } \times[C O]_{i n} / n(P t)
$$

Here, $\mathrm{CO}$ conversion rate is the percentage of $\mathrm{CO}$ oxidized to $\mathrm{CO}_{2}$ after the reaction. $[\mathrm{CO}]_{\text {in }}$ is the total molar flow of $\mathrm{CO}$ per second. $\mathrm{n}(\mathrm{Pt})$ stands for total moles of Pt atoms.

DFT calculations. Spin-polarized periodic DFT calculations were performed using the Vienna $\mathrm{Ab}$ initio Simulation Package ${ }^{43,44}$ to optimize the $\mathrm{Pt}_{4}$ clusters, $\mathrm{CO}$, $\mathrm{O}_{2}$ adsorptions and analyse the Bader charge of Pt/h-BNNS models. The $h$-BNNS was modelled using a $p(5 \times 5)$ supercell, with a vacuum of $20 \AA$ along the direction perpendicular to the substrate. The Generalized Gradient Approximation (GGA)PBE functiona ${ }^{45}$ was used for electronic exchange-correlation effect. The projector wave augmentation method ${ }^{46}$ was used to describe the electron-ion interaction, with a plane wave cutoff energy of $400 \mathrm{eV}$. A $4 \times 4 \times 1 \mathrm{k}$-points mesh based on the Monkhorst-Pack scheme ${ }^{41}$ was used for Brillouin-Zone integration. BEs on clean $h$-BNNS were calculated as: $\mathrm{BE}=\mathrm{E}_{\mathrm{Pt} 4 / h-\mathrm{BNNS}}-\mathrm{E}_{\mathrm{P} t 4}-\mathrm{E}_{\text {clean }} h$-BNNs; $\mathrm{BE}$ on Nv/Bv on $h$-BNNS are calculated as: $\mathrm{BE}=\mathrm{E}_{\mathrm{Pt} 4 \text { on Nv/Bv } h-\mathrm{BNNS})}-\mathrm{E}_{\mathrm{P} t 4}-\mathrm{E}_{\mathrm{Nv} / \mathrm{Bv}(h-\mathrm{BNNS})}$. 
a

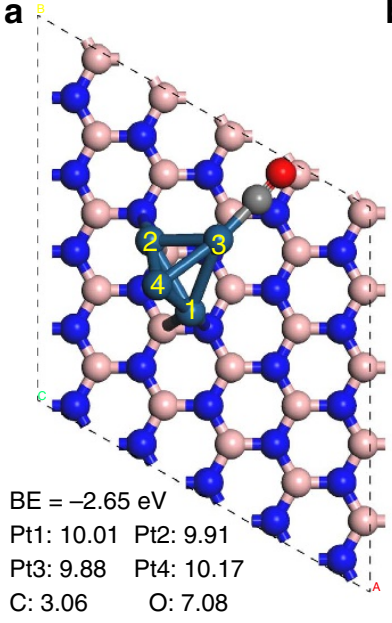

b

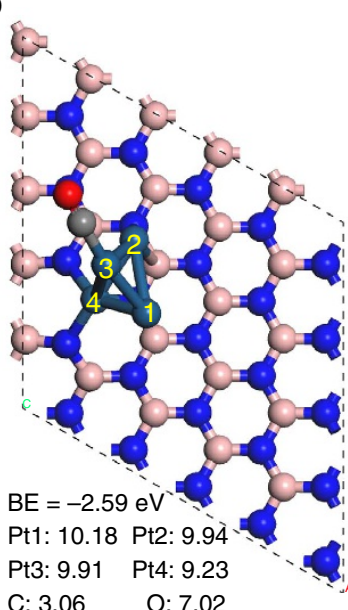

c.

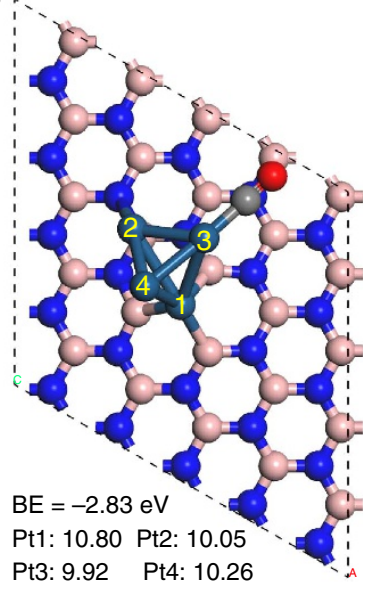

C: $3.04 \quad$ O: 7.09

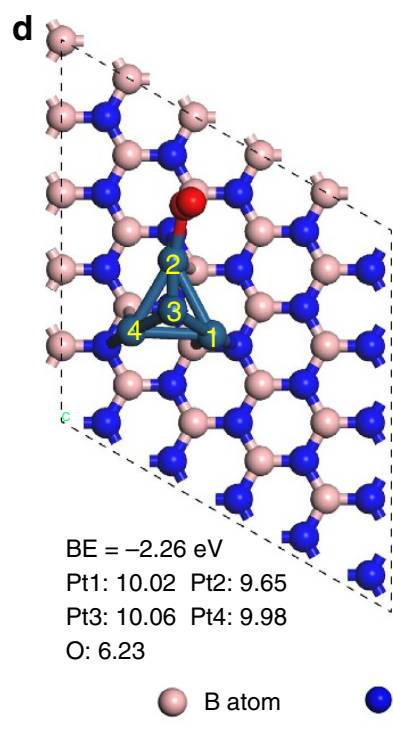

e

f

$\mathrm{BE}=-2.90 \mathrm{eV}$

Pt1: 10.00 Pt2: 10.01

Pt3: 9.58 Pt4: 9.19

O: 6.23
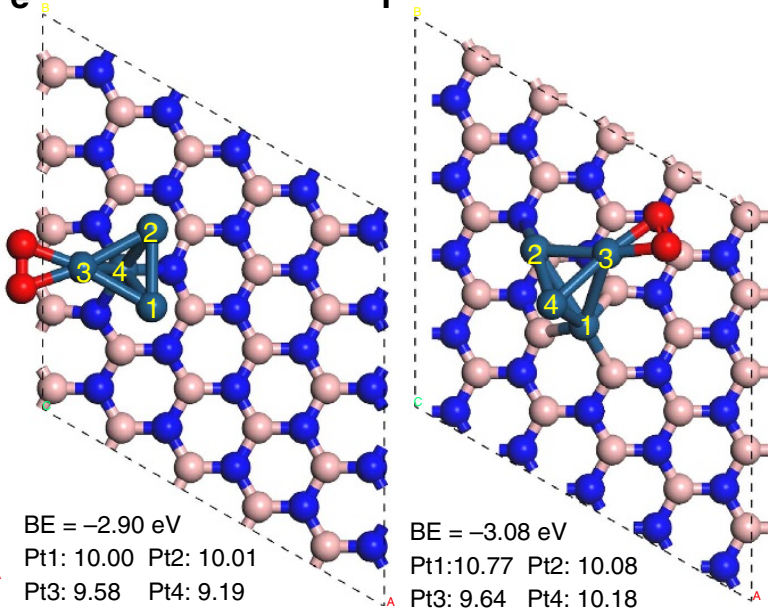

$0: 6.23$

$\mathrm{N}$ atom

Pt atom

C C atom

O atom

Figure 6 | Most stable configuration of $\mathbf{C O}$ and $\mathbf{O}_{\mathbf{2}}$ adsorption and binding energies on $\mathbf{P t}_{\mathbf{4}}$ cluster. $\mathbf{C O}$ adsorption: (a) $\mathrm{Pt}_{4}$ cluster on clean, vacancy-free $h$-BNNS. (b) $\mathrm{Pt}_{4}$ cluster $h$-BNNS with Bv. (c) Pt $t_{4}$ cluster on $h$-BNNS with Nv. $\mathrm{O}_{2}$ adsorption: (d) $\mathrm{Pt}_{4}$ cluster on clean, vacancy-free $h$-BNNS. (e) $\mathrm{Pt}_{4}$ cluster $h$-BNNS with Bv. (f) $\mathrm{Pt}_{4}$ cluster on $h$-BNNS with Nv.

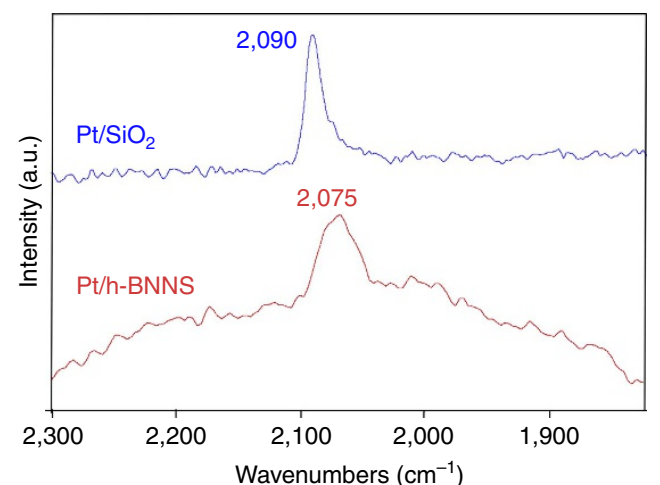

Figure 7 | In situ FTIR spectra of CO adsorbed on Pt/h-BNNS and $\mathbf{P t} / \mathrm{SiO}_{\mathbf{2}}$ at room temperature. Temperature $=25^{\circ} \mathrm{C}$. Features from gas phase $\mathrm{CO}$ have been subtracted.

Respective $\mathrm{BEs}$ of $\mathrm{CO}$ and $\mathrm{O}_{2}$ were calculated according to: $\mathrm{BE}_{\mathrm{CO} / \mathrm{O}^{*}}=\mathrm{E}_{\mathrm{CO} / \mathrm{O} 2^{*}}-$

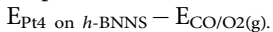

Data availability. The authors declare that the data supporting the findings of this study are available from the corresponding author on reasonable request.

\section{References}

1. Chen, G. X. et al. Interfacial electronic effects control the reaction selectivity of platinum catalysts. Nat. Mater. 15, 564-569 (2016).

2. Bai, Y. et al. Controllably interfacing with metal: a strategy for enhancing $\mathrm{CO}$ oxidation on oxide catalysts by surface polarization. J. Am. Chem. Soc. 136, 14650-14653 (2014).

3. Li, Q. et al. New approach to fully ordered fct-fept nanoparticles for much enhanced electrocatalysis in acid. Nano Lett. 15, 2468-2473 (2015).

4. Zhang, S., Metin, O., Su, D. \& Sun, S. H. Monodisperse AgPd alloy nanoparticles and their superior catalysis for the dehydrogenation of formic acid. Angew. Chem. Int. Ed. 52, 3681-3684 (2013).

5. Porter, N. S., Wu, H., Quan, Z. W. \& Fang, J. Y. Shape-control and electrocatalytic activity-enhancement of Pt-based bimetallic nanocrystals. Acc. Chem. Res. 46, 1867-1877 (2013).

6. Zhu, H. Y., Zhang, S., Guo, S. J., Su, D. \& Sun, S. H. Synthetic control of FePtM nanorods $(\mathrm{M}=\mathrm{Cu}, \mathrm{Ni})$ to enhance the oxygen reduction reaction. J. Am. Chem. Soc. 135, 7130-7133 (2013).

7. Mazumder, V., Chi, M. F., More, K. L. \& Sun, S. H. Core/Shell Pd/FePt nanoparticles as an active and durable catalyst for the oxygen reduction reaction. J. Am. Chem. Soc. 132, 7848-7849 (2010).

8. Zhang, S. et al. Monodisperse core/shell Ni/FePt nanoparticles and their conversion to $\mathrm{Ni} / \mathrm{Pt}$ to catalyze oxygen reduction. J. Am. Chem. Soc. 136, 15921-15924 (2014).

9. Zhang, L. et al. stabilization of palladium nanoparticles on nanodiamondgraphene core-shell supports for CO oxidation. Angew. Chem. Int. Ed. 54, 15823-15826 (2015). 
10. Zhu, H. et al. core/shell $\mathrm{Au} / \mathrm{MnO}$ nanoparticles prepared through controlled oxidation of AuMn as an electrocatalyst for sensitive $\mathrm{H}_{2} \mathrm{O}_{2}$ detection. Angew. Chem. Int. Ed. 53, 12508-12512 (2014).

11. Jiang, G. et al. Core/shell face-centered tetragonal FePd/Pd nanoparticles as an efficient non-Pt catalyst for the oxygen reduction reaction. ACS Nano 9, 11014-11022 (2015).

12. Wang, C., Xu, C., Zeng, H. \& Sun, S. Recent progress in syntheses and applications of dumbbell-like nanoparticles. Adv. Mater. 21, 3045-3052 (2009).

13. Jones, S., Qu, J., Tedsree, K., Gong, X. Q. \& Tsang, S. C. E. Prominent electronic and geometric modifications of palladium nanoparticles by polymer stabilizers for hydrogen production under ambient conditions. Angew. Chem. Int. Ed. 51, 11275-11278 (2012)

14. Cao, Z. et al. A molecular surface functionalization approach to tuning nanoparticle electrocatalysts for carbon dioxide reduction. J. Am. Chem. Soc. 138, 8120-8125 (2016).

15. Luksirikul, P., Tedsree, K., Moloney, M. G., Green, M. L. H. \& Tsang, S. C. E. Electron promotion by surface functional groups of single wall carbon nanotubes to overlying metal particles in a fuel-cell catalyst. Angew. Chem. Int. Ed. 51, 6998-7001 (2012).

16. Xiao, J. D. et al. Boosting photocatalytic hydrogen production of a metalorganic framework decorated with platinum nanoparticles: the platinum location matters. Angew. Chem. Int. Ed. 55, 9389-9393 (2016).

17. Stamenkovic, V. et al. Changing the activity of electrocatalysts for oxygen reduction by tuning the surface electronic structure. Angew. Chem. Int. Ed. 45, 2897-2901 (2006).

18. Stamenkovic, V. R. et al. Improved oxygen reduction activity on Pt3Ni(111) via increased surface site availability. Science 315, 493-497 (2007).

19. Chen, C. et al. Highly crystalline multimetallic nanoframes with threedimensional electrocatalytic surfaces. Science 343, 1339-1343 (2014).

20. Campbell, C. T. Catalyst-support interactions electronic perturbations. Nat. Chem 4, 597-598 (2012).

21. Kuo, C. H. et al. Understanding the role of gold nanoparticles in enhancing the catalytic activity of manganese oxides in water oxidation reactions. Angew. Chem. Int. Ed. 54, 2345-2350 (2015).

22. Tang, H. et al. Strong metal-support interactions between gold nanoparticles and nonoxides. J. Am. Chem. Soc. 138, 56-59 (2016).

23. Zhang, Y. H. et al. Hexagonal boron nitride cover on $\mathrm{Pt}(111)$ : a new route to tune molecule-metal interaction and metal-catalyzed reactions. Nano Lett. 15, 3616-3623 (2015)

24. Chen, G. X. et al. Interfacial effects in iron-nickel hydroxide-platinum nanoparticles enhance catalytic oxidation. Science 344, 495-499 (2014)

25. Giordano, L., Pacchioni, G., Noguera, C. \& Goniakowski, J. Identification of active sites in a realistic model of strong metal-support interaction catalysts: the case of Platinum (111)-supported iron oxide film. ChemCatChem 6, 185-190 (2014).

26. Guo, S. J., Zhang, S. \& Sun, S. H. Tuning nanoparticle catalysis for the oxygen reduction reaction. Angew. Chem. Int. Ed. 52, 8526-8544 (2013).

27. Wu, J. B. \& Yang, H. Platinum-based oxygen reduction electrocatalysts. Acc. Chem. Res. 46, 1848-1857 (2013).

28. Xu, Y. \& Zhang, B. Recent advances in porous Pt-based nanostructures: synthesis and electrochemical applications. Chem. Soc. Rev. 43, 2439-2450 (2014).

29. Zhu, H. Y. et al. Constructing hierarchical interfaces: $\mathrm{TiO}_{2}$-supported $\mathrm{PtFe}-$ FeOx nanowires for room temperature CO oxidation. J. Am. Chem. Soc. 137, 10156-10159 (2015)

30. Gao, Y. X., Wang, W. D., Chang, S. J. \& Huang, W. X. Morphology effect of $\mathrm{CeO}_{2}$ support in the preparation, metal-support interaction, and catalytic performance of $\mathrm{Pt} / \mathrm{CeO}_{2}$ catalysts. ChemCatChem 5, 3610-3620 (2013).

31. Vayssilov, G. N. et al. Support nanostructure boosts oxygen transfer to catalytically active platinum nanoparticles. Nat. Mater. 10, 310-315 (2011).

32. Qiao, B. et al. Single-atom catalysis of $\mathrm{CO}$ oxidation using $\mathrm{Pt} 1 / \mathrm{FeO}_{\mathrm{x}}$. Nat. Chem 3, 634-641 (2011).

33. Zhu, W. et al. Controlled gas exfoliation of boron nitride into few-layered nanosheets. Angew. Chem. Int. Ed. 55, 10766-10770 (2016).

34. Wang, C., Daimon, H., Onodera, T., Koda, T. \& Sun, S. A general approach to the size- and shape-controlled synthesis of platinum nanoparticles and their catalytic reduction of oxygen. Angew. Chem. Int. Ed. 47, 3588-3591 (2008).

35. Cretu, O. et al. Structure and local chemical properties of boron-terminated tetravacancies in hexagonal boron nitride. Phys. Rev. Lett. 114, 075502 (2015).

36. Suenaga, K., Kobayashi, H. \& Koshino, M. Core-level spectroscopy of point defects in single layer h-BN. Phys. Rev. Lett. 108, 075501 (2012).

37. Fu, Q. et al. Interface-confined ferrous centers for catalytic oxidation. Science 328, 1141-1144 (2010).

38. Mergler, Y. J., vanAalst, A., vanDelft, J. \& Nieuwenhuys, B. E. CO oxidation over promoted Pt catalysts. Appl. Catal. B-Environ. 10, 245-261 (1996).
39. Liu, X., Duan, T., Meng, C. G. \& Han, Y. Pt atoms stabilized on hexagonal boron nitride as efficient single-atom catalysts for $\mathrm{CO}$ oxidation: a firstprinciples investigation. RSC Adv 5, 10452-10459 (2015).

40. Lin, S., Ye, X. X., Johnson, R. S. \& Guo, H. First-Principles investigations of metal $(\mathrm{Cu}, \mathrm{Ag}, \mathrm{Au}, \mathrm{Pt}, \mathrm{Rh}, \mathrm{Pd}, \mathrm{Fe}, \mathrm{Co}$, and $\mathrm{Ir})$ doped hexagonal boron nitride nanosheets: stability and catalysis of CO Oxidation. J. Phys. Chem. C 117, 17319-17326 (2013).

41. Aal, S. A. CO catalytic oxidation on Pt-doped single wall boron nitride nanotube: first-principles investigations. Surf. Sci 644, 1-12 (2016).

42. Zhu, F. X., Wang, W. \& Li, H. X. Water-medium and solvent-free organic reactions over a bifunctional catalyst with au nanoparticles covalently bonded to $\mathrm{HS} / \mathrm{SO}_{3} \mathrm{H}$ functionalized periodic mesoporous organosilica. J. Am. Chem. Soc. 133, 11632-11640 (2011).

43. Kresse, G. \& Furthmuller, J. Efficiency of ab-initio total energy calculations for metals and semiconductors using a plane-wave basis set. Comp. Mat. Science $\mathbf{6}$, 15-50 (1996).

44. Kresse, G. \& Hafner, J. Ab initio molecular dynamics for open shell transitio metals. Phys. Rev. B 48, 13115-13118 (1993).

45. Perdew, J. P., Burke, K. \& Ernzerhof, M. Generalized gradient approximation made simple. Phys. Rev. Lett. 77, 3865-3868 (1996).

46. Monkhorst, H. J. \& Pack, J. D. Spe;cial points for Brillouin zone integrations. Phys. Rev. B 13, 5188-5192 (1976).

\section{Acknowledgements}

This work was supported by the US Department of Energy, Office of Science, Office of Basic Energy Sciences, Chemical Sciences, Geosciences and Biosciences Division Materials Science and Technology Division under contract number DE-AC0500OR22725. Part of the work including the IR and STEM study were conducted at the Center for Nanophase Materials Sciences, which is a DOE Office of Science User Facility. We thank the National Natural Science Foundation of China (Nos. 21576122 and 21376111 and a). A Project Funded by the Priority Academic Program Development of Jiangsu Higher Education Institutions (PAPD). The computational contribution was supported by the Start-up fund provided by Kansas State University; the National Science Foundation under Award No. EPS-0903806, and matching support from the State of Kansas through the Kansas Board of Regents for financial support. M.Z. and B.L. also acknowledge the supercomputing resources provided by the Center for Nanoscale Materials (CNM) of the Office of Science of the US Department of Energy under the contract No. DE-AC02-06CH11357; the Beocat Research Cluster at Kansas State University, which is funded in part by NSF grants CNS-1006860; and the National Energy Research Scientific Computing Center (NERSC) under the contract No. DE-AC02-05CH11231.

\section{Author contributions}

W.Z., H.Z. and S.D. conceived the idea. W.Z. prepared and characterized the catalysts and performed the CO catalytic oxidation experiment. Z.W. and G.S.F. performed in situ IR spectroscopy measurements. X.G. and H.N.L. carried out STEM and EELS. G.M.V. and K.L.B. provided XPS. M.Z., B.L. and H.L. provided theoretical calculation and discussion. W.Z. and H.Z. wrote the paper. P.W., Z.W. and S.D. discussed the results and participated in the preparation of the paper.

\section{Additional information}

Supplementary Information accompanies this paper at http://www.nature.com/ naturecommunications

Competing interests: The authors declare no competing financial interests.

Reprints and permission information is available online at http://npg.nature.com/ reprintsandpermissions/

How to cite this article: Zhu, W. et al. Taming interfacial electronic properties of platinum nanoparticles on vacancy-abundant boron nitride nanosheets for enhanced catalysis. Nat. Commun. 8, 15291 doi: 10.1038/ncomms15291 (2017).

Publisher's note: Springer Nature remains neutral with regard to jurisdictional claims in published maps and institutional affiliations.

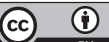

This work is licensed under a Creative Commons Attribution 4.0 International License. The images or other third party material in this article are included in the article's Creative Commons license, unless indicated otherwise in the credit line; if the material is not included under the Creative Commons license, users will need to obtain permission from the license holder to reproduce the material. To view a copy of this license, visit http://creativecommons.org/licenses/by/4.0/

\footnotetext{
C) The Author(s) 2017
} 